\section{AK117, A CD47 BLOCKING ANTIBODY WITH ROBUST MACROPHAGE ACTIVATION WITHOUT RED BLOOD CELL HEMAGGLUTINATION}

'Zhaoliang Huang, 'Xinghua Pang, 'Tingting Zhong, 'Tailong Qu, 'Chunshan Jin, ${ }^{1} \mathrm{NA}$ Chen*, ${ }^{1}$ Xinrong He, ${ }^{1}$ Dennis Xia, ${ }^{2}$ Xiaoping Jin, ${ }^{1} Z$ Zhongmin Wang, ${ }^{1} \mathrm{Xu}$ Xia, ${ }^{1}$ Baiyong Li. ' Akeso Biopharma Inc., Zhongshan, China; ${ }^{2}$ Akeso Biopharma Inc, Potomac, USA

Background AK117 is a humanized monoclonal antibody targeting CD47 which widely expresses on innate immune cells, such as macrophages, and functions as a regulator of phagocytosis. CD47 serves as the ligand for a receptor on these innate immune cells, SIRPo, which in turn delivers an inhibitory signal for phagocytosis. Hematology toxicity is the major concern of an anti-CD47 antibody. As an agent targeting CD47 being investigated as an anti-tumor therapeutic, AK117 is engineered on a human IgG4 scaffold to minimize recruitment of Fc-dependent effector functions, as well as identified with favorable hematology safety profile and robust pro-phagocytosis activity.

Methods Activity of AK117 binding to CD47 to block the interaction between CD47 and SIRP $\alpha$ were determined by FACS, and binding of AK117 to human RBC was also evaluated. Raji cells, HT-29 cells, and HL-60 cells which highly express CD47 were used as target cells to evaluate a prophagocytic activity of AK117 as a monotherapy or in combination with anti-EGFR antibody, anti-CD20 antibody or azacitidine. In in-vivo pharmacology studies, anti-tumor activity of AK117 was investigated in SCID/beige mouse Raji tumor model. Effects of AK117 on hemagglutination of human RBC at was tested. Hemoglobin (HGB) and hematocrit (HCT) was evaluated after single dose of $10 \mathrm{mg} / \mathrm{kg}$ AK117 or Hu-5F9 in male and female cynomolgus monkeys ( $n=1 /$ gender).

Results AK117 could effectively binds to CD47, and competes with SIRP $\alpha$ for binding to the antigen on Raji cells (figure 1). AK117 alone or combines with anti-EGFR antibody, antiCD20 antibody and azacitidine shows potent phagocytosis of tumor cells in a dose-dependent manner (figure 2). AK117 significantly inhibited tumor growth in these tumor models (figure 3). Favorable hematology safety profile of AK117 was observed. A significant weaker binding to human RBC of AK117 was identified (figure 4), and AK117 does not induce hemagglutination of human $\mathrm{RBC}$ up to a concentration of $1050 \mu \mathrm{g} / \mathrm{mL}$, while $\mathrm{Hu}-5 \mathrm{~F} 9$ triggers hemagglutination even at a low concentration of $1.44 \mu \mathrm{g} / \mathrm{mL}$ (figure 5). AK117 has minimal anemia effect in monkey studies compared to hu5F9G4 after single dose in cynomolgus monkeys (figure 6). AK117 showed a rather superior safety profile to Hu5F9-G4 as a shorter duration of anemia.
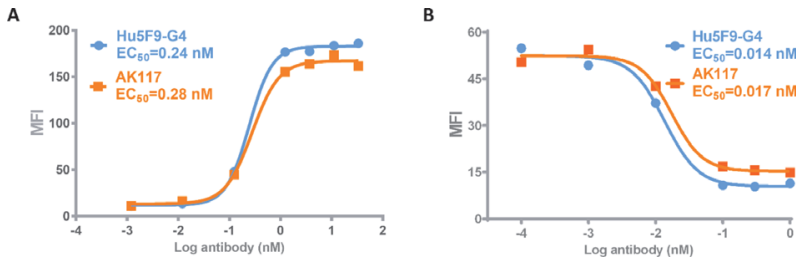

Abstract 266 Figure 1 Binding and Competition activity of AK117 to CD47. (A) FACS binding curves of AK117 and Hu5F9-G4 to CD47 on raji cells. (B) FACS competitive binding curve of AK117 and Hu5F9-G4 with SIRP $\alpha E C D-m F c$ to CD47 on raji cells.
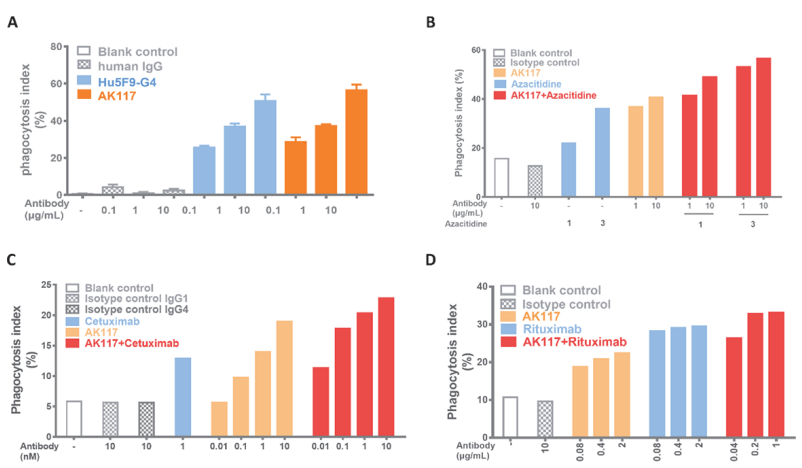

Abstract 266 Figure 2 The pro-phagocytic activity against tumor cells. (A) The phagocytic index of raji cells by macrophages with AK117. (B) The phagocytic index of HL-60 cells by macrophages with AK117 and azacitidine. (C) The phagocytic index of HT-29 cells by macrophages with AK117 and cetuximab. (D) The phagocytic index of raji cells by macrophages with AK117 and rituximab.
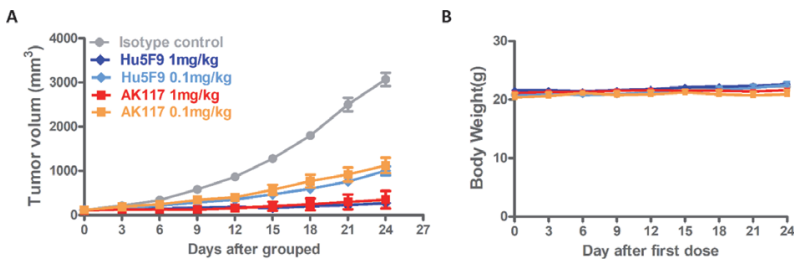

Abstract 266 Figure 3 Anti-tumor activity in raji tumor mouse model. The (A) Tumor growth curves and (B) Body weight curves of different groups in SCID/Beige mice with subcutaneous raji tumor.

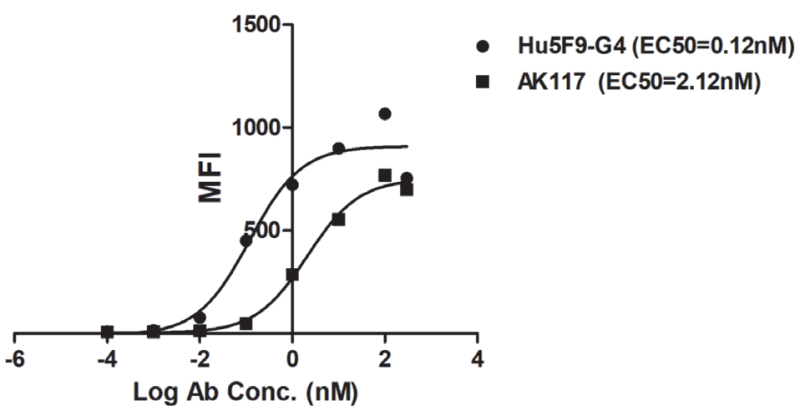

Abstract 266 Figure 4 Binding activity of AK117 to human RBCs. Binding Curves of Hu5F9-G4 and AK117 to CD47 on human RBCS

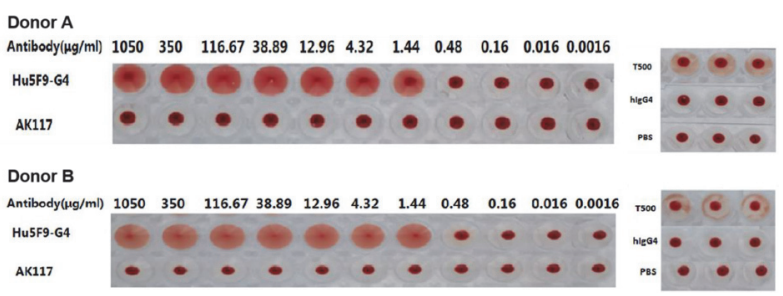

Abstract 266 Figure 5 Hemagglutination effect on human erythrocytes. Hemagglutination effect of AK117 on human erythrocytes 
A

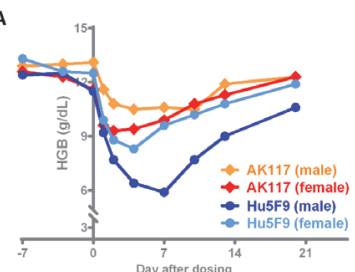

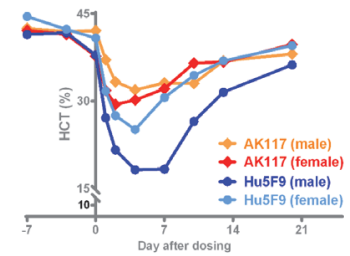

Abstract 266 Figure 6 HGB and HCT in cynomolgus monkeys. The curves of (A) hemoglobin and (B) Hematocrit at different times in cynomolgus monkeys.

Conclusions With pre-clinical pharmacology activities comparable to Hu5F9-G4 as well as superior safety properties demonstrated in non-clinical pharmacodynamics studies, AK117 has emerged as a promising new treatment for solid tumor.

http://dx.doi.org/10.1136/jitc-2021-SITC2021.266 